\title{
THE SET OF ALL $m \times n$ RECTANGULAR REAL MATRICES OF RANK $r$ IS CONNECTED BY ANALYTIC REGULAR ARCS
}

\author{
J.-Cl. EVARD AND F. JAFARI
}

(Communicated by Palle E. T. Jorgensen)

\begin{abstract}
It is well known that the set of all square invertible real matrices has two connected components. The set of all $m \times n$ rectangular real matrices of rank $r$ has only one connected component when $m \neq n$ or $r<m=n$. We show that all these connected components are connected by analytic regular arcs. We apply this result to establish the existence of $p$-times differentiable bases of the kernel and the image of a rectangular real matrix function of several real variables.
\end{abstract}

\section{INTRODUCTION}

In [3] we showed that every open connected subset of a topological vector space is connected by regular polynomial curves. In this paper, we deal with the set of real $m \times n$ matrices of rank $r$. In spite of the fact that this set is not an open subset of $\mathbb{R}^{m \times n}$, we show that it is connected by regular arcs that are not only of class $C^{\infty}$ but are also analytic. A similar result was established in [2, Theorem 7.2] for complex matrices, but some new methods are necessary to obtain arcs that are contained in the set of real matrices. We furnish a method to construct these arcs explicitly.

The analytic connections are likely to have many applications. For example, a method to construct continuous arcs in the set of square invertible real matrices is furnished in [1, Proposition 1.5]. This construction was used to establish the uniqueness of the topological degree. In this paper, we provide another application by showing that the main result of [2] about the existence of bases of class $C^{p}$ of the kernel and the image of a rectangular matrix function of several real variables is also valid when the field is $\mathbb{R}$ instead of $\mathbb{C}$.

We will denote by $\mathbb{R}^{m \times n}$ the set of all $m \times n$ real matrices and by $\mathbb{R}_{r}^{m \times n}$ the subset of $\mathbb{R}^{m \times n}$ of all matrices of rank $r$. We will denote by $I_{n}$ the $n \times n$ identity matrix and by $I_{r}^{m \times n}$ the following $m \times n$ matrix of rank $r$ :

$$
I_{r}^{m \times n}=\left[\begin{array}{cc}
I_{r} & 0 \\
0 & 0
\end{array}\right] .
$$

In Lemma 1 we show that the two connected components of $\mathbb{R}_{n}^{n \times n}$ are connected by analytic arcs that may be chosen as closed curves travelled infinitely

Received by the editors May 29, 1992.

1991 Mathematics Subject Classification. Primary 15A54, 54D05. 
many times. In Lemma 2 we establish the existence of equivalences with positive determinants between matrices of same rank. In Lemma 3 we show that $\mathbb{R}_{r}^{m \times n}$ is connected by analytic arcs that may be chosen as closed curves travelled infinitely many times, when $m \neq n$ or $r<m=n$. In Theorem 4 we show that all the connected components of $\mathbb{R}_{n}^{n \times n}$ and $\mathbb{R}_{r}^{m \times n}$ are connected by analytic arcs that are regular. The problem of finding analytic regular closed curves travelled infinitely many times is still open. In Theorem 5 we apply Theorem 4 to establish the existence of bases of class $C^{p}$ of the kernel and the image of a rectangular real matrix function of several real variables.

\section{RESULTS}

Lemma 1. Let $A, B \in \mathbb{R}_{n}^{n \times n}$ be such that $\operatorname{det} A$ and $\operatorname{det} B$ have the same sign. Then there exists an analytic mapping $F: \mathbb{R} \rightarrow \mathbb{R}_{n}^{n \times n}$ such that, for every $m \in \mathbb{Z}$, $F(m)=A$ if $m$ is even and $F(m)=B$ if $m$ is odd. Moreover, $\operatorname{det} F(t)$ has the same sign as $\operatorname{det} A$, and $F(t+2)=F(t)$ for every $t \in \mathbb{R}$.

Proof. Let $C=B A^{-1} \in \mathbb{R}_{n}^{n \times n}$. Since $\operatorname{det} A$ and $\operatorname{det} B$ have the same sign, we have $\operatorname{det} C>0$. It is well known that $C$ is similar in $\mathbb{R}^{n \times n}$ to a real Jordan matrix. More precisely, there exist $R \in \mathbb{R}_{n}^{n \times n}$ and $J \in \mathbb{R}^{n \times n}$ such that $C=R J R^{-1}$, where $J$ has the form

$$
J=\operatorname{diag}\left[J_{1}, J_{2}, J_{3}\right],
$$

where in turn $J_{1}$ has the form

$$
\begin{aligned}
J_{1} & =\left[\begin{array}{cccc}
C\left(\rho_{1}, \theta_{1}\right) & \alpha_{1} I_{2} & & 0 \\
& & \ddots & \\
& \ddots & & \alpha_{p-1} I_{2} \\
& & & C\left(\rho_{p}, \theta_{p}\right)
\end{array}\right], \\
\rho_{1}, \ldots, \rho_{p}>0, \theta_{1}, \ldots, \theta_{p} \in[0,2 \pi[, & \alpha_{1}, \ldots, \alpha_{p-1} \in\{0,1\}, \\
C(\rho, \theta) & =\rho\left[\begin{array}{cc}
\cos \theta & \sin \theta \\
-\sin \theta & \cos \theta
\end{array}\right] \quad \forall \rho>0, \theta \in[0,2 \pi[;
\end{aligned}
$$

$J_{2}$ has the form

$$
J_{2}=\left[\begin{array}{cccc}
\mu_{1} & \beta_{1} & & 0 \\
& & \ddots & \\
& \ddots & & \beta_{q-1} \\
0 & & & \mu_{q}
\end{array}\right],
$$

$\mu_{1}, \ldots, \mu_{q}>0, \beta_{1}, \ldots, \beta_{q-1} \in\{0,1\} ;$ and $J_{3}$ has the form

$$
J_{3}=\left[\begin{array}{cccc}
v_{1} & \gamma_{1} & & 0 \\
& \cdot & \ddots & \\
& \ddots & & \gamma_{r-1} \\
0 & & & v_{r}
\end{array}\right] \text {, }
$$

$v_{1}, \ldots, v_{r}<0, \gamma_{1}, \ldots, \gamma_{r-1} \in\{0,1\}$, and $r$ is even because $\operatorname{det} C>0$. Let

$$
\lambda(t)=\cos ^{2}(\pi t / 2), \quad \mu(t)=\sin ^{2}(\pi t / 2) \quad \forall t \in \mathbb{R} .
$$


Let $t \in \mathbb{R}$. For every $k \in\{1,2, \ldots\}$ that makes sense, let

$$
\begin{array}{cc}
r_{k}(t)=\lambda(t)+\mu(t) \rho_{k}, \quad s_{k}(t)=\mu(t) \theta_{k}, & a_{k}(t)=\mu(t) \alpha_{k}, \\
C_{k}(t)=C\left(r_{k}(t), s_{k}(t)\right), & \\
m_{k}(t)=\lambda(t)+\mu(t) \mu_{k}, & b_{k}(t)=\mu(t) \beta_{k}, \\
n_{k}(t)=-\lambda(t)+\mu(t) v_{k}, & c_{k}(t)=\mu(t) \gamma_{k} .
\end{array}
$$

Let

$$
\begin{aligned}
& H_{1}(t)=\left[\begin{array}{cccc}
C_{1}(t) & a_{1}(t) I_{2} & & 0 \\
& \ddots & & a_{p-1}(t) I_{2} \\
& \ddots & & C_{p}(t)
\end{array}\right] \\
& H_{2}(t)=\left[\begin{array}{cccc}
m_{1}(t) & b_{1}(t) & & 0 \\
& & \ddots & \\
& \ddots & & b_{q-1}(t) \\
0 & & & m_{q}(t)
\end{array}\right] \text {, } \\
& R(t)=\left[\begin{array}{cc}
\cos (\pi t) & \sin (\pi t) \\
-\sin (\pi t) & \cos (\pi t)
\end{array}\right], \\
& H_{3}(t)=\left[\begin{array}{cccc}
n_{1}(t) & c_{1}(t) & & 0 \\
& & \ddots & \\
& \ddots & & c_{r-1}(t) \\
0 & & & n_{r}(t)
\end{array}\right]\left[\begin{array}{ccc}
-R(t) & & 0 \\
& \ddots & \\
0 & & -R(t)
\end{array}\right],
\end{aligned}
$$

where the last matrix has $\frac{r}{2}$ blocks $R(t)$, which is possible since $r$ is even.

$$
\begin{gathered}
H(t)=\operatorname{diag}\left[H_{1}(t), H_{2}(t), H_{3}(t)\right], \\
G(t)=R H(t) R^{-1}, \quad F(t)=G(t) A .
\end{gathered}
$$

Let $m \in \mathbb{Z}$. Plainly, if $m$ is even, then

$$
\begin{gathered}
H_{1}(m)=I_{2 p}, \quad H_{2}(m)=I_{q}, \quad H_{3}(m)=\left(-I_{r}\right)\left(-I_{r}\right)=I_{r}, \\
H(m)=I_{n}, \quad G(m)=R R^{-1}=I_{n}, \quad F(m)=I_{n} A=A,
\end{gathered}
$$

and, if $m$ is odd, then

$$
\begin{gathered}
H_{1}(m)=J_{1}, \quad H_{2}(m)=J_{2}, \quad H_{3}(m)=J_{3}, \\
H(m)=J, \quad G(m)=R J R^{-1}=C, \quad F(m)=C A=B .
\end{gathered}
$$

It is obvious that all the functions above are periodic with period 2. Let $t \in \mathbb{R}$.

It is easy to see that

$$
\begin{aligned}
& \operatorname{det} H_{1}(t)=\operatorname{det} C_{1}(t) \cdots \operatorname{det} C_{p}(t)=\left(r_{1}(t)\right)^{2} \cdots\left(r_{p}(t)\right)^{2}>0, \\
& \operatorname{det} H_{2}(t)=m_{1}(t) \cdots m_{q}(t)>0, \\
& \operatorname{det} H_{3}(t)=n_{1}(t) \cdots n_{r}(t)(\operatorname{det} R(t))^{r / 2}=(-1)^{r}\left|n_{1}(t)\right| \cdots\left|n_{r}(t)\right|>0,
\end{aligned}
$$


because $r$ is even, and, finally,

$$
\begin{aligned}
& \operatorname{det} H(t)=\left(\operatorname{det} H_{1}(t)\right)\left(\operatorname{det} H_{2}(t)\right)\left(\operatorname{det} H_{3}(t)\right)>0, \\
& \operatorname{det}(G(t))=\operatorname{det} H(t)>0, \\
& \operatorname{det} F(t)=(\operatorname{det} G(t))(\operatorname{det} A) \neq 0 .
\end{aligned}
$$

Thus $F(t) \in \mathbb{R}_{n}^{n \times n}$, and $\operatorname{det} F(t)$ has the same sign as $\operatorname{det} A$.

Lemma 2. Let $m, n \in\{1,2, \ldots\}$ and $r \in\{0,1,2, \ldots\}$ be such that $m \neq n$ or $r<m=n$. Let $A \in \mathbb{R}_{r}^{m \times n}$. Then there exist $L \in \mathbb{R}_{m}^{m \times m}$ and $R \in \mathbb{R}_{n}^{n \times n}$. such that $\operatorname{det} L>0$, $\operatorname{det} R>0$, and $A=L I_{r}^{m \times n} R$.

Proof. If $r=0$, then $A=0=I_{r}^{m \times n}$, and we can choose $L=I_{m}$ and $R=I_{n}$. Suppose $r \geq 1$. It follows from the hypothesis that $r<m$ or $r<n$.

(a) Suppose $r<m$. Then $m \geq 2$. As $A$ is of rank $r$, it is well known that $A$ is equivalent in $\mathbb{R}_{r}^{m \times n}$ to $I_{r}^{m \times n}$. That is, there exist $B \in \mathbb{R}_{m}^{m \times m}$ and $C \in \mathbb{R}_{n}^{n \times n}$ such that $A=B I_{r}^{m \times n} C$. If $\operatorname{det} B>0$ and $\operatorname{det} C>0$, we obviously choose $L=B$ and $R=C$. If $\operatorname{det} B>0$ and $\operatorname{det} C<0$, we choose

$$
L=B \operatorname{diag}\left[-1, I_{m-2},-1\right] \quad \text { and } R=\operatorname{diag}\left[-1, I_{n-1}\right] C \text {, }
$$

considering that $r=n$ is possible. If $\operatorname{det} B<0$ and $\operatorname{det} C>0$, we choose

$$
L=B \operatorname{diag}\left[I_{m-1},-1\right] \text { and } R=C \text {. }
$$

If $\operatorname{det} B<0$ and $\operatorname{det} C<0$, we choose

$$
L=B \operatorname{diag}\left[-1, I_{m-1}\right] \quad \text { and } \quad R=\operatorname{diag}\left[-1, I_{n-1}\right] C \text {. }
$$

It is easy to check that, in all cases, $A=L I_{r}^{m \times n} R, \operatorname{det} L>0$, and $\operatorname{det} R>0$.

(b) Suppose $r<n$. Then by (a), there exist $B \in \mathbb{R}_{n}^{n \times n}$ and $C \in \mathbb{R}_{m}^{m \times m}$ such that $A^{\top}=B I_{r}^{n \times m} C$, $\operatorname{det} B>0$, and $\operatorname{det} C>0$. Let $L=C^{\top}$ and $R=B^{\top}$. Then

$$
\begin{gathered}
A=C^{\top} I_{r}^{m \times n} B^{\top}=L I_{r}^{m \times n} R, \\
\operatorname{det} L=\operatorname{det} C^{\top}=\operatorname{det} C>0, \quad \operatorname{det} R=\operatorname{det} B^{\top}=\operatorname{det} B>0 .
\end{gathered}
$$

Lemma 3. Let $m, n \in\{1,2, \ldots\}$ and $r \in\{0,1, \ldots\}$ be such that $m \neq n$ or $r<m=n$. Let $A, B \in \mathbb{R}_{r}^{m \times n}$. Then there exists an analytic mapping $F: \mathbb{R} \rightarrow \mathbb{R}_{r}^{m \times n}$ such that, for every $k \in \mathbb{Z}, F(k)=A$ if $k$ is even and $F(k)=B$ if $k$ is odd. Moreover, $F(t+2)=F(t)$ for every $t \in \mathbb{R}$.

Proof. By Lemma 2 there exist $A_{1}, B_{1} \in \mathbb{R}_{m}^{m \times m}$ and $A_{2}, B_{2} \in \mathbb{R}_{n}^{n \times n}$ such that

$$
\begin{array}{rrlr}
A=A_{1} I_{r}^{m \times n} A_{2}, & B=B_{1} I_{r}^{m \times n} B_{2}, \\
\operatorname{det} A_{1}>0, \quad \operatorname{det} A_{2}>0, & \operatorname{det} B_{1}>0, \quad \operatorname{det} B_{2}>0 .
\end{array}
$$

By Lemma 1 there exist analytic mappings $F_{1}: \mathbb{R} \rightarrow \mathbb{R}_{m}^{m \times m}$ and $F_{2}: \mathbb{R} \rightarrow \mathbb{R}_{n}^{n \times n}$ such that, for every $k \in \mathbb{Z}, F_{1}(k)=A_{1}, F_{2}(k)=A_{2}$ if $k$ is even and $F_{1}(k)=$ $B_{1}, F_{2}(k)=B_{2}$ if $k$ is odd. Let

$$
F(t)=F_{1}(t) I_{r}^{m \times n} F_{2}(t) \quad \forall t \in \mathbb{R} .
$$

Then $F: \mathbb{R} \rightarrow \mathbb{R}_{r}^{m \times n}$ is analytic, and, for every $k \in \mathbb{Z}, F(k)=A_{1} I_{r}^{m \times n} A_{2}=A$ if $k$ is even and $F(k)=B_{1} I_{r}^{m \times n} B_{2}=B$ if $k$ is odd.

Theorem 4. The subset $\mathbb{R}_{n}^{n \times n}$ of $\mathbb{R}^{n \times n}$ has two connected components, whereas the subset $\mathbb{R}_{r}^{m \times n}$ of $\mathbb{R}^{m \times n}$ has only one connected component when $m \neq n$ or 
$r<m=n$. When $r>0$, all these connected components are connected by analytic regular arcs. More precisely: Suppose $r>0$. Let $A, B \in \mathbb{R}_{r}^{m \times n}$ be such that $A \neq B$, and, if $r=m=n$, then $\operatorname{det} A$ and $\operatorname{det} B$ have the same sign. Then there exists an analytic mapping $F: \mathbb{R} \rightarrow \mathbb{R}_{r}^{m \times n}$ such that $F(0)=A$, $F(1)=B$, and $F^{\prime}(t) \neq 0$ for every $t \in[0,1]$.

Proof. By Lemma 1 (if $r=m=n$ ) and Lemma 3 (if $m \neq n$ or $r<m=$ $n)$, there exists an analytic mapping $G: \mathbb{R} \rightarrow \mathbb{R}_{r}^{m \times n}$ such that $G(0)=A$ and $G(1)=B$. Let

$$
\chi=\left\{t \in[0,1] \mid \exists \lambda(t) \in \mathbb{R}, G^{\prime}(t)=\lambda(t) G(t)\right\} .
$$

Case 1: Suppose $\chi$ is infinite. Then there exist $t_{1}, t_{2}, \ldots \in \chi$ and $t_{0} \in[0,1]$ such that $t_{0}=\lim _{k \rightarrow \infty} t_{k}$. For every $t \in \mathbb{R}, i \in\{1, \ldots, m\}, j \in\{1, \ldots, n\}$, let $g_{i j}(t)$ denote the entry of the $i$ th row, $j$ th column of $G(t)$. Since $r>0$, there exist $i_{0} \in\{1, \ldots, m\}$ and $j_{0} \in\{1, \ldots, n\}$ such that $g_{i_{0} j_{0}}\left(t_{0}\right) \neq 0$. As $g_{i_{0} j_{0}}$ is continuous, there exists a neighborhood $N_{t_{0}} \subseteq \mathbb{R}$ of $t_{0}$ such that $g_{i_{0} j_{0}}(t) \neq 0$ for every $t \in N_{t_{0}}$. Because $\lim _{k \rightarrow \infty} t_{k}=t_{0}$, there exists $k_{0} \in \mathbb{N}$ such that $t_{k} \in N_{t_{0}}$ for every $k \in\left\{k_{0}, k_{0}+1, \ldots\right\}$. Let $k \in\left\{k_{0}, k_{0}+1, \ldots\right\}$. Since $t_{k} \in \chi$, we have $G^{\prime}\left(t_{k}\right)=\lambda\left(t_{k}\right) G\left(t_{k}\right)$, which implies $g_{i_{0} j_{0}}^{\prime}\left(t_{k}\right)=\lambda\left(t_{k}\right) g_{i_{0} j_{0}}\left(t_{k}\right)$ and, hence, $g_{i_{0} j_{0}}\left(t_{k}\right) G^{\prime}\left(t_{k}\right)=g_{i_{0} j_{0}}^{\prime}\left(t_{k}\right) G\left(t_{k}\right)$. As $\lim _{k \rightarrow \infty} t_{k}=t_{0}$, it follows by the Analytic Continuation Theorem that $g_{i_{0} j_{0}}(t) G^{\prime}(t)=g_{i_{0} j_{0}}^{\prime}(t) G(t)$ for every $t \in \mathbb{R}$. Let $g=g_{i_{0} j_{0}}$. The equality $g G^{\prime}-g^{\prime} G=0$ implies that $(G(t) / g(t))^{\prime}=0$ for every $t \in N_{t_{0}}$. Therefore, there exists a constant matrix $M_{0} \in \mathbb{R}^{m \times n}$ such that $G(t)=g(t) M_{0}$ for every $t \in N_{t_{0}}$ and hence for every $t \in \mathbb{R}$ by analytic continuation. Because $\operatorname{rank} G=r>0$, the equality $G=g M_{0}$ implies that $g(t) \neq 0$ for every $t \in \mathbb{R}$ and $M_{0} \neq 0$. Furthermore, $A=G(0)=g(0) M_{0}$ and $B=G(1)=g(1) M_{0}$. Let

$$
F(t)=\{t(g(1)-g(0))+g(0)\} M_{0} \quad \forall t \in \mathbb{R} .
$$

Then

$$
F(0)=g(0) M_{0}=A, \quad F(1)=g(1) M_{0}=B,
$$

and

$$
F^{\prime}(t)=(g(1)-g(0)) M_{0} \neq 0,
$$

for every $t \in \mathbb{R}$, because $A \neq B$ implies that $g(0) \neq g(1)$.

Case 2: Suppose that $\chi$ is finite. Then there exist $t_{0}, \ldots, t_{q} \in[0,1]$ such that $0=t_{0}<t_{1}<\cdots<t_{q}=1$ and

$$
\chi \subseteq\left\{t_{0}, \ldots, t_{q}\right\} .
$$

By Hermite interpolation, there exists a polynomial $p \in \mathbb{R}[X]$ such that, for every $k \in\{0, \ldots, q\}$,

$$
\begin{gathered}
p\left(t_{k}\right)=\frac{1}{2}, \\
t_{k} \in \chi \text { and } \lambda\left(t_{k}\right) \neq 0 \Rightarrow p^{\prime}\left(t_{k}\right)=\lambda\left(t_{k}\right), \\
t_{k} \in \chi \text { and } \lambda\left(t_{k}\right)=0 \Rightarrow p^{\prime}\left(t_{k}\right)=1 .
\end{gathered}
$$

For every $t \in \mathbb{R}$, let

$$
q(t)=p(t)^{2}+\frac{3}{4}, \quad F(t)=q(t) G(t) .
$$


Then, by (2), $F(0)=G(0)=A$ and $F(1)=G(1)=B$. Moreover, for every $t \in \mathbb{R}$, we have $\operatorname{rank} F(t)=\operatorname{rank} G(t)=r$, because $q(t) \neq 0$. Let $t \in[0,1]$. Let us show that $F^{\prime}(t) \neq 0$. Suppose $F^{\prime}(t)=0$. Then

$$
0=F^{\prime}(t)=q^{\prime}(t) G(t)+q(t) G^{\prime}(t) .
$$

Consequently,

$$
G^{\prime}(t)=-\frac{q^{\prime}(t)}{q(t)} G(t),
$$

which implies that $t \in \chi$. It follows by (1), (2), (3), (4) that

$$
\begin{gathered}
q(t)=p(t)^{2}+\frac{3}{4}=1, \\
q^{\prime}(t)=2 p(t) p^{\prime}(t)=p^{\prime}(t)= \begin{cases}\lambda(t) & \text { if } \lambda(t) \neq 0, \\
1 & \text { if } \lambda(t)=0 .\end{cases}
\end{gathered}
$$

On the other hand, since $t \in \chi$, we have

$$
G^{\prime}(t)=\lambda(t) G(t)
$$

and, hence, by (5),

$$
0=\left(q^{\prime}(t)+q(t) \lambda(t)\right) G(t) .
$$

Since $r>0$, we have $G(t) \neq 0$, and it follows that

$$
q^{\prime}(t)+q(t) \lambda(t)=0 .
$$

Consequently, by (6) and (7),

$$
0=\lambda(t)+\lambda(t)=2 \lambda(t) \quad \text { if } \lambda(t) \neq 0
$$

and

$$
0=1+1 \cdot 0=1 \quad \text { if } \lambda(t)=0 .
$$

Both cases are impossible. Therefore, $F^{\prime}(t) \neq 0$ for every $t \in[0,1]$.

Theorem 5 (Existence of orthonormal bases of class $C^{p}$ of the kernel and the image of a rectangular matrix function of $q$ real variables). Let $\Omega \subseteq \mathbb{R}^{q}$ be $C^{p}$-diffeomorphic to $\mathbb{R}^{q}$. Let $A \in C^{p}\left(\Omega, \mathbb{R}_{r}^{m \times n}\right)$. Then there exist

$$
u_{1}, \ldots, u_{m} \in C^{p}\left(\Omega, \mathbb{R}^{m}\right), \quad v_{1}, \ldots, v_{n} \in C^{p}\left(\Omega, \mathbb{R}^{n}\right)
$$

such that, for every $t \in \Omega$,

(a) if $r>0$, then $\left(u_{1}(t), \ldots, u_{r}(t)\right)$ is an orthonormal basis of $\operatorname{Im} A(t)$;

(b) if $r<m$, then $\left(u_{r+1}(t), \ldots, u_{m}(t)\right)$ is an orthonormal basis of $(\operatorname{Im} A(t))^{\perp}$

(c) if $r>0$, then $\left(v_{1}(t), \ldots, v_{r}(t)\right)$ is an orthonormal basis of $(\operatorname{Ker} A(t))^{\perp}$;

(d) if $r<n$, then $\left(v_{r+1}(t), \ldots, v_{n}(t)\right)$ is an orthonormal basis of $\operatorname{Ker} A(t)$.

Proof. The proof is the same as the proof of Theorem 8.2 of [2] except for the following modifications:

(a) Replace $\mathbb{C}$ by $\mathbb{R}$ in the proof of Theorem 8.2 of [2].

(b) In the proof of Lemma 8.1 of [2], apply Theorem 4 of this paper instead of Theorem 7.2 of [2].

(c) In the proof of Lemma 8.1 of [2], if $\operatorname{det} X(t)<0$, then multiply the first column of $A(t)$ and $X(t)$ by -1 . 


\section{REFERENCES}

1. K. Deimling, Nonlinear functional analysis, Springer, New York, 1985.

2. J.-Cl. Evard, On the existence of bases of class $C^{p}$ of the kernel and the image of a matrix function, Linear Algebra Appl. 135 (1990), 33-67.

3. J.-Cl. Evard and F. Jafari, Polynomial path connectedness and Hermite interpolation in topological vector spaces, submitted.

Department of Mathematics, University of Wyoming, LaRAmie, Wyoming 82071-3036 E-mail address, J.-Cl. Evard: matdifeq@corral .uwyo.edu

E-mail address, F. Jafari: f jafari@corral.uwyo.edu 